\title{
Design of Composite Laminates Containing Pin Loaded Holes
}

\author{
FU-Kuo Chang AND Richard A. ScOTT \\ Department of Mechanical Engineering and Applied Mechanics \\ The University of Michigan, Ann Arbor, Michigan 48109
}

AND

GEORGE S. SPRINGER

Department of Aeronautics and Astronautics

Stanford University, Stanford, California 94305

(Received November 11, 1983)

(Revised February 23, 1984)

\begin{abstract}
Methods are presented for sizing composite laminates containing one, two, or several pin loaded holes, the holes being arranged either in a single row or in two parallel rows. It is shown how the design parameters (number of holes, hole diameter, hole positions) can be found, which result in the maximum failure load and in the maximum failure load per unit weight.
\end{abstract}

\section{INTRODUCTION}

N THE PRECEEDING PAPER [1], A METHOD WAS DESCRIBED FOR CALCULATling the failure strengths and failure modes of laminates containing either one or two pin loaded holes. In this paper, it is shown how the model (together with the "Bolt" computer code [1]) can be used to size laminates containing more than two pin loaded holes.

It is desired to determine the number of holes, the hole diameter, and the hole positions which result in the maximum failure load $P_{M}$ and the maximum failure load per unit weight $\mathbf{P}_{\mathbf{M}}^{*}$. The failure load per unit weight is defined as

$$
P^{*}=P / w
$$

where $P$ is the failure load, and $w$ is the combined weight of the composite $w_{c}$ and the pin(s) $w_{s}$

$$
w=w_{c}+w_{s}
$$

Journal of COMPOSITE MATERIALS, Vol. 18-May 1984 
In this paper, procedures suitable for calculating $\mathrm{P}_{\mathrm{M}}$ and $\mathrm{P}_{\mathrm{M}}^{*}$ are illustrated via two sample problems. In these problems, the failure load of 24-ply (thickness $\mathrm{H}=0.125$ in) $\left[(0 / \pm 45 / 90)_{3}\right]_{\text {s }}$ Fiberite T300/1034-C graphite-epoxy composites are determined. The material properties used in calculations are listed in Table 1 . The density of the composite is $\varrho_{c}=0.00194 \mathrm{lbm} / \mathrm{in}^{3}$. The pin or pins are assumed to be $3 / 4$ in long and to be made of steel (density $Q_{s}=$ $0.0093 \mathrm{lbm} / \mathrm{in}^{3}$ ).

The calculation procedures are presented in Section 3 for joints containing one or two holes, and in Section 4 for joints containing three or more holes. First, however, interferences between two adjacent holes, between the edge and an adjacent hole, and between the side and an adjacent hole are discussed.

\section{INTERACTION COEFFICIENTS}

It is desired to know under what conditions, if any, the proximity of two holes, or the proximity of a hole to the edge or to the side of the laminate, affects the failure load. The interaction between two holes, between a hole and the edge, and between a hole and the side, can best be evaluated by the use of interaction coefficients.

\section{Two Holes in Parallel}

The parallel hole interaction coefficient $g_{H}$ is defined by

$$
g_{H}=P_{s} /\left(P_{H} / 2\right)
$$

where $P_{s}$ is the failure load of a laminate of width $G_{H}$ containing a single loaded hole, and $P_{H}$ is the failure load of a laminate of width $2 \mathrm{G}_{\mathrm{H}}$ containing two loaded holes separated by a distance $G_{H}$ (Figure 1 ). When $G_{H}$ becomes large, the interaction between two holes becomes small $\left(P_{H} / 2 \rightarrow P_{s}\right)$ and the interaction coefficient approaches unity.

Table 1. Properties of fiberite T300/1034C graphite/epoxy composite.

\begin{tabular}{llc}
\hline Longitudinal Young's Modulus, $E_{x}$ & $=21300000 \mathrm{psi}$ \\
Transverse Young's Modulus, $E_{y}$ & $=1700000 \mathrm{psi}$ \\
Shear Modulus, $G_{x y}$ & $=897000 \mathrm{psi}$ \\
Poisson's Ratio $\mu_{x y}$ & $=0.3$ \\
Longitudinal Tensile Strength, $X_{t}$ & $=251000 \mathrm{psi}$ \\
Longitudinal Compressive Strength, $X_{c}=200000 \mathrm{psi}$ \\
Rail Shear Strength, $S$ & $=19400 \mathrm{psi}$ \\
Characteristic Length in Tension, $\mathrm{R}_{\mathrm{t}}$ & $=0.018 \mathrm{in}$ \\
Characteristic Length in Compression, $\mathrm{R}_{\mathrm{c}}$ & $=0.07 \mathrm{in}$ \\
\hline
\end{tabular}




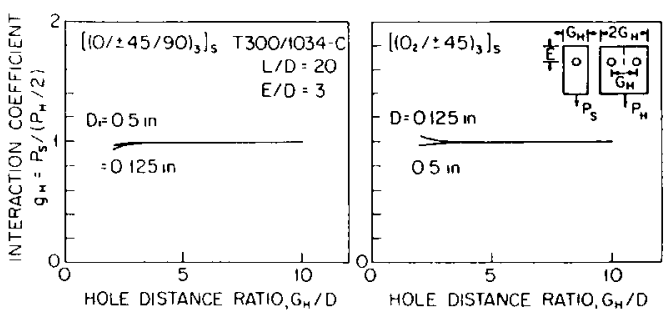

Figure 1. Interaction coefficient for two holes in parallel.

\section{Two Holes in Series}

The series hole interaction coefficient $g_{v}$ is defined by

$$
g_{V}=P_{V} / P_{T}
$$

where $P_{V}$ is the failure load of a laminate (width W) containing two loaded holes separated by a distance $G_{v}$. $P_{T}$ is the failure load of a laminate with the same width containing two loaded holes; one located at a distance $E$ from the edge, and the other located at the center of the laminate (Figure 2). When the hole separation distance $G_{v}$ increases, the influence of one hole on the other becomes small; the failure load $P_{V}$ approaches $P_{T}\left(P_{V} \rightarrow P_{T}\right)$ and $g_{V}$ approaches unity.

\section{Edge Interaction}

The edge interaction coefficient $g_{E}$ is defined by

$$
g_{E}=P_{s} / P_{C}
$$

where $P_{s}$ is the failure load of a laminate (width W) with a single-loaded hole
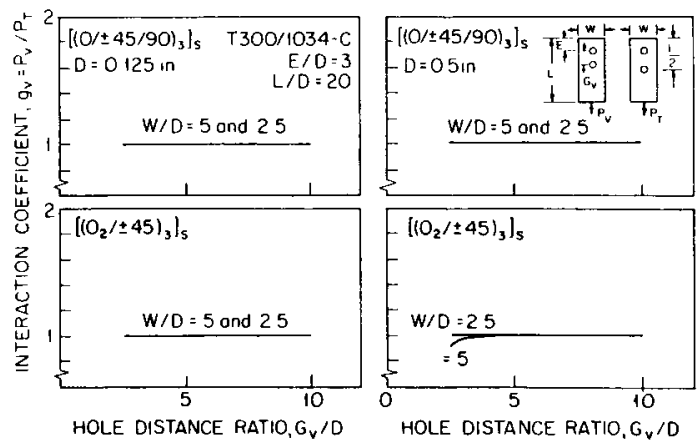

Figure 2. Interaction coefficient for two holes in series. 
at a distance $E$ from the edge. $P_{c}$ is the failure load of a laminate of width $W$ with a loaded hole in the center (Figure 3 ). The influence of the edge on the failure load becomes smaller as the edge distance increases. When the hole is moved to the center $(\mathrm{E}=\mathrm{L} / 2), P_{s}$ becomes $P_{C}$ and the interaction coefficient becomes unity.

\section{Side Interaction Coefficient}

The side interaction coefficient $g_{s}$ is defined by

$$
g_{s}=P_{H} / P_{G}
$$

where $P_{H}$ is the failure load of a laminate (width W) containing two loaded holes separated by a distance $\mathrm{W} / 2 . P_{G}$ is the failure load of a laminate with the same width containing two loaded holes separated by a distance $G_{H}\left(G_{H} \geqslant\right.$ $\mathrm{W} / 2$, Figure 4)*. As the distance $Q$ between the side and the hole increases $P_{G}$ approaches $P_{H}$ and the interaction coefficient $g_{s}$ approaches unity.

\section{Numerical Values of the Interaction Coefficients}

In order to illustrate trends in the interaction coefficients, these coefficients were calculated for Fiberite T300/1034-C graphite-epoxy composite laminates with ply orientations of $\left.\left[(0 / \pm 45 / 90)_{3}\right)\right]_{s}$ and $\left[\left(0_{2} / \pm 45\right)_{3}\right]_{s}$. The results, obtained using the Bolt computer code, are presented in Figures 1-4. The most significant feature of these results is that the failure load is not affected significantly:

a) by the proximity of two holes in parallel when the distance between two holes is larger than $3 \mathrm{D}$,

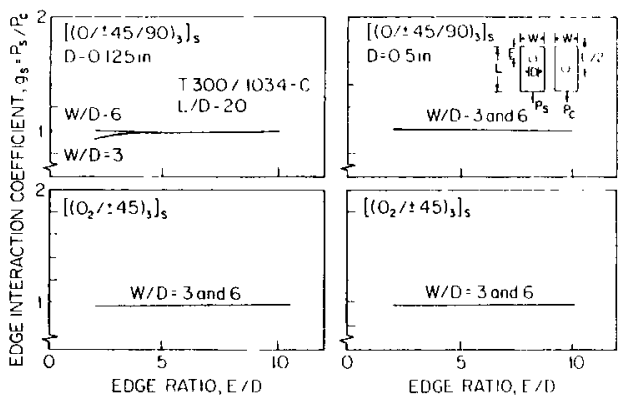

Figure 3. Edge interaction coefficient.

"Dashed lines in the Figures indicate areas in the code "BOLT" that cannot be computed, at least with the current version of the automatic mesh generator. 

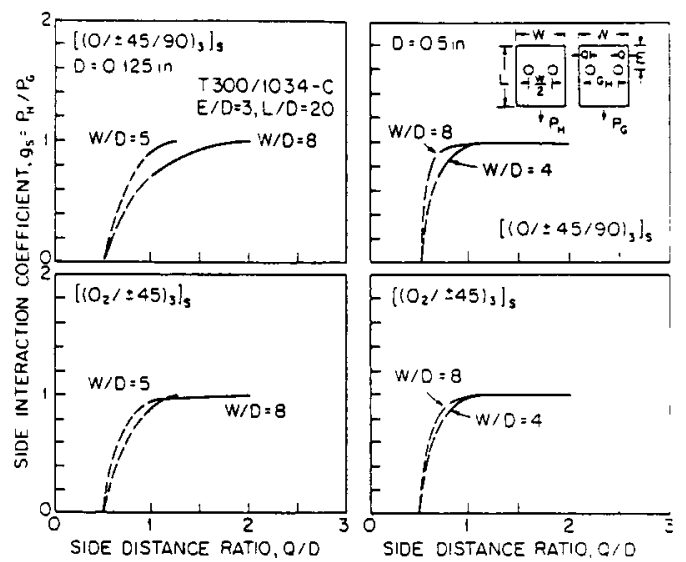

Figure 4. Side interaction coefficient.

b) by the proximity of two holes in series when the distance between two holes is larger than $2 \mathrm{D}$,

c) by the edge when the distance between the edge and the hole is greater than 3D,

d) by the proximity of side when the distance between the hole and the side is larger than 2D.

Mathematically, these conditions can be expressed as

$$
\begin{array}{lr}
g_{H} \rightarrow I P_{H} \rightarrow 2 P_{s} \text { for } G_{H} / D \geqslant 3 & \\
g_{V} \rightarrow I P_{V} \rightarrow P_{T} \text { for } G_{V} / D \geqslant 2 & \text { for }[(0 / \pm 45 / 90)]_{s} \\
g_{E} \rightarrow I P_{S} \rightarrow P_{C} \text { for } E / D \geqslant 3 & {\left[\left(0_{2} / \pm 45\right)_{3}\right]_{s}} \\
g_{S} \rightarrow I P_{G} \rightarrow P_{H} \text { for } Q / D \geqslant 2 &
\end{array}
$$

It should be emphasized that the conditions expressed by the relations (7) may not apply for every ply orientiation. The conditions at which the different coefficients become unity must be evaluated separately for each laminate lay up.

\section{DESIGN OF LAMINATES WITH ONE OR TWO HOLES}

In this section, a procedure is described which can be used to size a laminate containing either one or two pin-loaded holes.

We consider a laminate of known width ( $W=1$ in), length ( $L=8$ in) and thickness $(\mathrm{H}=0.125$ in). The laminate may contain either one pin-loaded hole or two pin-loaded holes in parallel or in series, as illustrated in Figure 5.

It is desired to find the number of holes (one or two holes), the hole diameter $\mathrm{D}$, the edge distance $E$ and the distance between two holes $G$, which 

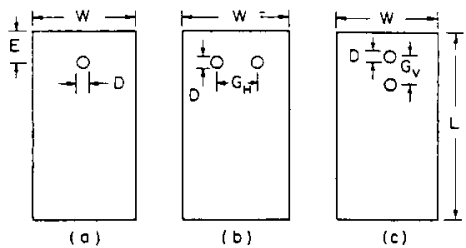

Figure 5. Description of the problem used in designing laminates with al single pin-loaded hole, b) two pin-loaded holes in parallel, cl two pin-loaded holes in series.

result in the maximum failure load $\mathrm{P}_{\mathrm{M}}$ and in the maximum failure load per unit weight $\mathrm{P}_{\mathrm{M}}^{*}$.

The calculations, performed with the "BOLT" computer code [1], proceed along the following major steps:

a) The failure loads of a laminate containing a single loaded hole are calculated for different hole diameters $\mathrm{D}$ and for different edge distance ratios $\mathrm{E} / \mathrm{D}$. The failure load is plotted versus the edge ratio $\mathrm{E} / \mathrm{D}$ (Figure 6). The desired edge ratio (E/D) is selected.

Here, the edge ratio $E / D=3$ is selected because the failure load reaches a maximum at an edge ratio of about 3 and remains nearly constant at higher edge ratios. This value $(E / D=3)$ will also be used for joints containing two loaded holes in parallel and two holes in series. The reasons for this choice of E/D in the multihole case are as follows: 1) For parallel holes the interaction between two holes has almost no effect on the failure load $\left(g_{H} \rightarrow I\right.$ and $P_{H} / 2 \rightarrow P_{S}$, Section 2). 2) For two holes in series, the interaction between the holes is unimportant when $G_{v} / D>3\left(g_{v} \rightarrow 1\right.$ and $P_{v} \rightarrow P_{c}$, Section 2).

b) The failure loads are calculated for different hole diameters and for different hole separations $G$ for two holes in parallel and for two holes in

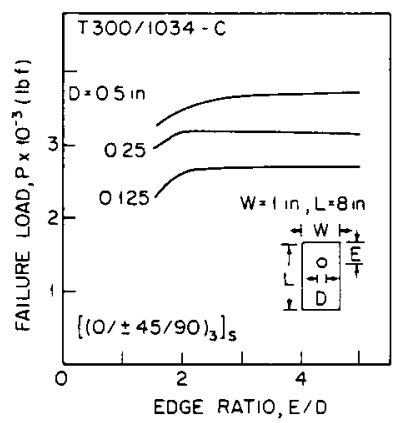

Figure 6. Failure load as a function of edge ratio for laminates containing a single pin-loaded hole. 
series. The failure loads are plotted as functions of the hole distance ratio $G / D$ (Figure 7, top). From these plots, the maximum failure load $\mathrm{P}_{\mathrm{M}}$ can be obtained.

For the problem under consideration, the maximum failure load is $5000 \mathrm{lb}$. This load is achieved with two 0.125 in diameter pins in parallel separated by a distance $G_{H}=0.5$ in $\left(G_{H} / D=4\right)$.

c) From the known values of the failure load $P$, the failure load per unit weight $P^{*}$ is calculated using the expression

$$
P^{*}=P /\left[\varrho_{\mathrm{c}} W H L+a\left(\pi D^{2} / 4\right)\left(\mathrm{Q}_{s} L,-\varrho_{\mathrm{e}} H\right)\right] g
$$

where $L_{s}$ is the length of the pin. The parameter a is $a=1$ for a single hole, $a=\mathbf{2}$ for two holes. The failure load per unit weight is plotted as a function of $G / D$ (Figure 7, bottom).

For the present problem, the maximum failure load per unit weight $\mathrm{P}_{\mathrm{M}}^{*}$ is $8,000 \mathrm{lbf} / \mathrm{lbf}$ and occurs with two 0.125 in diameter pins separated by a horizontal distance $G_{H}=0.5$ in $\left(G_{H} / D=4\right)$.

\section{DESIGN OF LAMINATES WITH MULTIPLE HOLES}

This problem is concerned with laminates containing several pin-loaded holes spaced evenly, either in a single row or in two parallel rows, as illustrated in Figure 8.

The number of holes in the laminate with a single row of holes, or the number of columns in the laminates with two rows of holes is (for the special case in which the distance from the side to the hole is $G_{H} / 2$ )

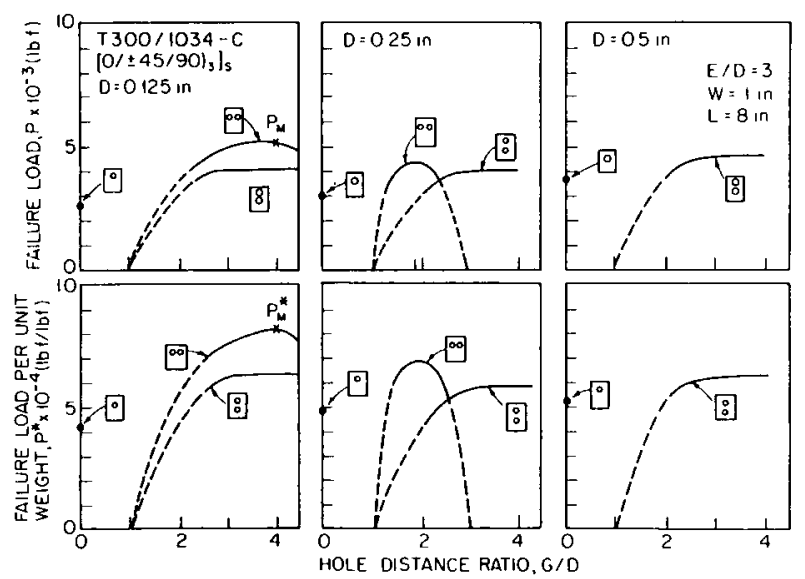

Figure 7. Failure load (top) and failure load per unit weight (bottom) of laminates containing a single pin-loaded hole. Two pin-loaded holes in parallel and two pin-loaded holes in series. 

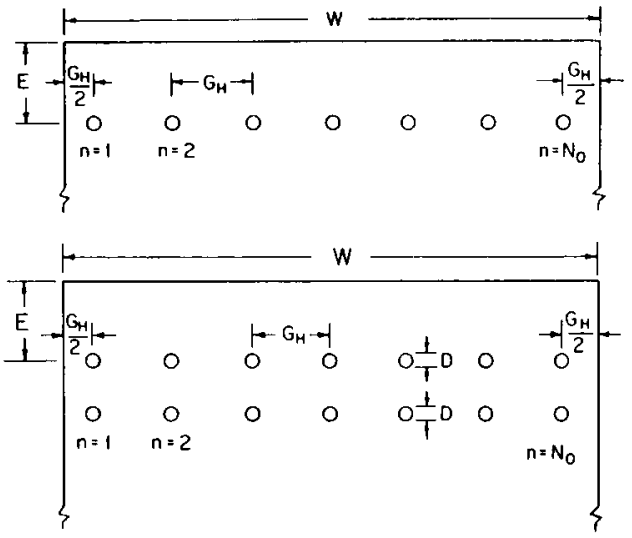

Figure 8. Geometry of single row of holes (top) and two rows of holes (bottom).

$$
N_{\circ}=W / G_{H}
$$

It is desired to determine the number of holes $N_{o}$, the hole size $D$, the positions of the holes $G_{H}$ and $G_{V}$, and the edge distance $E$ which result in the maximum failure load.

The analytical method described in Reference [1] together with the "BOLT" code can be applied only to laminates containing either a single pinloaded hole, or two pin-loaded holes in parallel or in series. Therefore, this method cannot be used directly to calculate the failure load of a laminate containing several holes. The failure loads of such laminates can still be estimated with the use of this method by the procedure described below.

a) The interaction coefficients $g_{E}$ and $g_{V}$ are calculated and plotted in exactly the same way as was described in Section 2.

b) The ratios $E / D$ and $G_{v} / D$ are selected which correspond to the conditions $g_{E} \rightarrow 1$ and $g_{v} \rightarrow 1$. In this investigation, the values of both $E / D$ and $G_{v} / D$ were selected to be 3 because both $g_{E}$ and $g_{v}$ reach unity at this ratio. This $E / D$ ratio is used for a single row of holes. This is also a reasonable choice for two rows of holes because, to a large degree, the first row of holes acts independently of the second row of holes.

c) Values are assumed for the number of holes and the hole diameter. The distance $G_{H}$ is calculated from

$$
G_{H}=W / N_{\circ}
$$

d) The failure load is calculated for a $2 G_{H}$ wide laminate containing two loaded holes in parallel, $P_{H}$, and for a laminate of width $G_{H}$ containing two loaded holes in series, $P_{V}$. The interaction parameters $g_{H}$ and $g_{s}$ are then calculated for the geometry under consideration, according to the method given in Section 2. 
e) The failure load is approximated by the expression

$$
P=P_{N_{0}-2}+2 P_{\text {side }}
$$

where $P_{N_{0}-2}$ is the load carried by the second $(\mathrm{n}=2)$ through the next to last $\left(\mathrm{n}=N_{0}-1\right)$ pins, and $P_{\text {stde }}$ is the load carried by the first $(\mathrm{n}=1)$ and last $\left(\mathrm{n}=N_{\mathrm{o}}\right)$ pins. Thus, the failure load of a laminate containing one or two rows of holes is

$$
\begin{array}{ll}
P_{r 1}=\left(\left(N_{o}-2\right) / 2\right) g_{H} P_{H}+g_{s} P_{H} & \text { (one row) } \\
P_{r 2}=\left(N_{o}-2\right) g_{H}^{2} P_{V}+2 g_{H} g_{S} P_{V} & \text { (two rows) }
\end{array}
$$

where the subscripts $r 1$ and $r 2$ refer to one row or two rows of holes, respectively $(a=1,2)$.

If $\mathrm{Q}=G_{H} / 2$ then $g_{s}$ is equal to unity. This is the case in the present problem. Accordingly,

$$
\begin{aligned}
& P_{r 1}=\left(\left(N_{o}-2\right) / 2\right) g_{H} P_{H}+P_{H} \\
& P_{r 2}=\left(N_{o}-2\right) g_{H}^{2} P_{V}+2 g_{H} P_{V}
\end{aligned}
$$

f) The failure load per unit weight $P^{*}$ is calculated from

$$
P_{r l, r 2}^{*}=p_{r l, r 2} /\left[\mathrm{e}_{c} W H L+a N_{\mathrm{o}}\left(\pi D^{2} / 4\right)\left(\mathrm{Q}_{s} L_{s}-\mathrm{Q}_{\mathrm{c}} H\right)\right] g
$$

g) The calculations are repeated for different values of $N_{o}$ and $D$. The failure load $P_{r l, r 2}$ and the failure load per unit weight $P_{r 1, r 2}^{*}$ are plotted as functions of $N_{o}$. From these figures, the maximum failure load $P_{M}^{*}$ and the maximum failure load per unit weight $\mathbf{P}_{\mathrm{M}}^{*}$ are determined.

In the present problem a 4 in wide and 10 in long composite laminate was considered. The ply orientation is $\left.[0 / \pm 45 / \pm 90)_{3}\right]_{s}$. The foregoing procedure gave the maximum failure load $P_{M}=23,400 \mathrm{lbf}$ when there are twelve 0.125 in diameter holes arranged in two rows of holes (Figure 9). The maximum failure load per unit weight $\left(\mathrm{P}_{\mathrm{M}}^{*}=67,000 \mathrm{lbf} / \mathrm{lbf}\right)$ is achieved with twelve 0.125 in diameter holes in a single row (Figure 9).

\section{Failure Mode}

The results generated by the computer code also show the modes of failure. The changes in the modes of failure with the number of holes $N_{\circ}$ are illustrated in Figure 10. In the present sample problem, at the condition of the maximum failure load $\left(N_{0}=12\right)$ the failure mode is in tension. Failure in such a mode often happens quite suddenly. In some situations it might be preferable to choose a design in which failure occurs by a less sudden failure mode. For example, failure would have occurred in bearing mode if, in the 

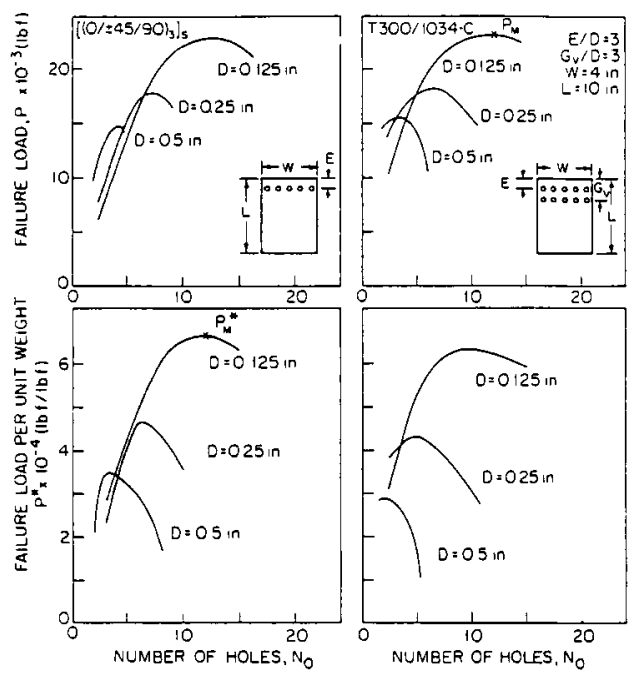

Figure 9. Failure load (top) and failure load per unit weight (bottom) of laminates containing one row (left) and two rows (right) of pin-loaded holes.

present problem, a hole diameter of 0.125 in were chosen, and the number of holes were taken to be $N_{0}=6$. However, this would have resulted in a 30 to 40 percent reduction in the failure load.

\section{CONCLUDING REMARKS}

The method described in this paper can be used to size laminates containing one or more pin loaded holes. The method can also be applied to the design of bolted joints provided the bolt tightness does not significantly affect the strength of the joint. This is frequently the case, as was discussed in Reference [1].
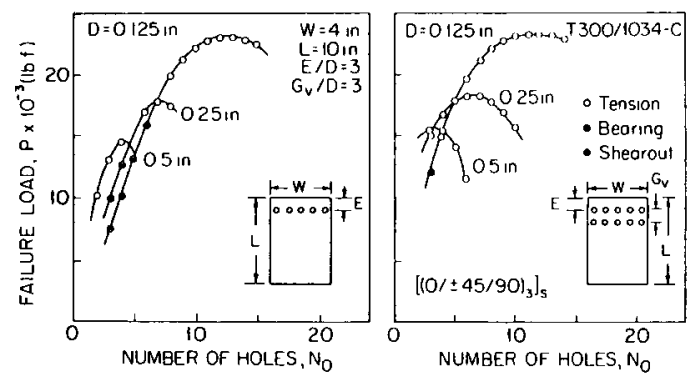

Figure 10. Failure modes of single row (left) and two rows (right) of pin-loaded holes. 


\section{ACKNOWLEDGEMENTS}

This work was supported by the Mechanics and Surface Interactions Branch, Nonmetallic Materials Division, Materials Laboratory, Air Force Wright Aeronautical Laboratories, Wright-Patterson AFB, Ohio. Dr. S. W. Tsai was the project engineer.

\section{REFERENCE}

1. Chang, F. K., Scott, R. A., and Springer, G. S., "Failure of Composite Laminates Containing Pin Loaded Holes-Method of Solution," Vol. 18, pp. 255-278 (1984). 